\title{
COMMON MODE VOLTAGE SUPPRESSION METHOD BASED ON MODEL PREDICTIVE CONTROL FOR A PERMANENT MAGNET SYNCHRONOUS MOTOR CONSIDERING CURRENT ERROR LIMIT
}

\author{
XIAOFAN WANG, XIAOCHUN FANG, FEI LIN \& ZHONGPING YANG \\ School of Electrical Engineering, Beijing Jiaotong University, China
}

\begin{abstract}
The common mode voltage (CMV) generated by the inverter will lead to bearing damage and electromagnetic interference issues. This paper studies the CMV suppression method based on finite control set model predictive control (FCS-MPC) for permanent magnet synchronous motor (PMSM) drive fed by voltage source inverter. The advantage of the method proposed in this paper is that it balances both current quality assurance and CMV suppression. The balance of the current quality and CMV suppression can be achieved by adjusting a single parameter. Meanwhile, the method can maintain low switching frequency. The performance of the proposed method was evaluated by computer simulation and compared with other methods.

Keywords: model predictive control, permanent magnet synchronous motor, common mode voltage, current quality, low switching frequency.
\end{abstract}

\section{INTRODUCTION}

In recent years, the permanent magnet synchronous motor (PMSM) drive system is increasingly used in rail transit. PMSM is generally driven by the voltage source inverter, as is shown in Fig. 1. The common mode voltage (CMV) generated by the inverter not only brings the problem of electromagnetic interference, but also generates shaft voltage on the motor shaft. When the shaft voltage is greater than the bearing grease insulation voltage threshold, the bearing will produce destructive shaft current, resulting in bearing raceway groove, increase bearing friction, the bearing life is shortened, affecting the safe operation of the drive system [1]. Therefore, in order to reduce the negative impact of CMV, CMV suppression issue has received wide attention from scholars.

Existing pulse width modulation (PWM) based CMV suppression methods include active zero state pulse width modulation (active zero state PWM, AZSPWM) technology [2], remote three vector pulse width modulation (remote state PWM, RSPWM) technology [3] (Near state PWM, NSPWM) technology [4], etc., but these methods require complex PWM module.

Finite control set model predictive control (FCS-MPC) has the advantages of fast response, flexible design and no PWM. In [5], the CMV suppression is studied based on FCSMPC. This method removes two zero vectors, leaving only six non-zero vectors as candidates. References [6] and [7] use the idea of deadbeat control to achieve CMV suppression. Although this method can reduce the computational burden, but the differential part will produce a lot of noise. Reference [8] designed the voltage suppression algorithm using two non-zero voltage vectors during each sampling period to achieve CMV suppression and to reduce total harmonic distortion (THD) of the current. However, the algorithm needs to optimize the selection of two vectors, which leads to computational complexity and high switching frequency. In reference [9], in order to reduce the switching frequency, switching between non-adjacent voltage vectors is prohibited, and therefore, there are only four candidate voltage vectors. (In this paper, we call this method as Conventional 4VVs). Based 


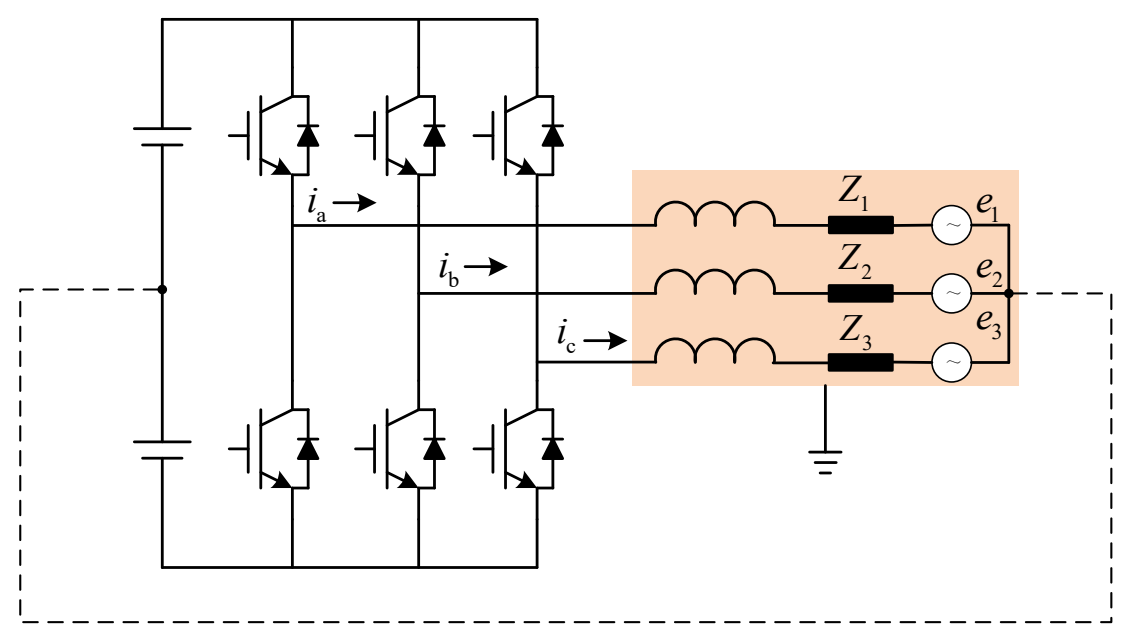

Figure 1: Topology of the PMSM drive system.

on the Conventional 4VVs method, reference [10] proposed to abandon the zero vector and use a non-adjacent vector as the fourth candidate so that CMV can be suppressed. (In this paper, we call this method as Non-zero 4VVs). The disadvantage of this method is the increase of the switching frequency and the lack of flexibility.

In this paper, based on the Conventional 4VVs scheme, we designed a more flexible approach for CMV suppression. The current quality is taken as an important consideration, and the balance between the current quality and the CMV suppression can be adjusted. M, the switching frequency will remain low. Subsequent chapters are arranged as following: In Section 2, we establish a discrete model of traction inverters and PMSMs and describe the predictive current control framework using a simplified control set. In Section 3, we designed the CMV suppression method considering current error limit. In Section 4, the simulation results show the performance of the proposed method and the switching frequency. Section 5 is the conclusion.

\section{FCS-MPC FOR PMSM}

The basic idea of FCS-MPC is to use the discrete model of the motor drive. Because the traction inverter is made up of a limited number of power devices, it has a finite number of switching states, that is, they form a finite control set. Then, an optimal switching state is selected by predicting the values of the state variables at the next sampling moment. So we can send a switching command directly to the inverter without PWM process.

\subsection{Discrete time modelling}

The discrete predictive model of PMSM in synchronous rotating coordinate is as follows:

$$
\left[\begin{array}{c}
i_{d}{ }^{p}(k+1) \\
i_{q}{ }^{p}(k+1)
\end{array}\right]=A_{k}\left[\begin{array}{c}
i_{d}(k) \\
i_{q}(k)
\end{array}\right]+B_{k}\left[\begin{array}{l}
u_{d}(k) \\
u_{q}(k)
\end{array}\right]+E_{k},
$$

where $i_{d}$ and $i_{q}$ are the $d$-axis and $q$-axis components of the stator current, $u_{d}$ and $u_{q}$ are the $d$ axis and $q$-axis components of the stator voltage, $x(k)$ represents the variable $x$ corresponding 
to $k, x(k+1)$ represents the variable $x$ corresponding to $k+1$, the superscript $p$ represents the predicted value of the corresponding variable. The coefficient matrices $A_{k}, B_{k}$ and the back electromotive force $E_{k}$ are as follows:

$$
\begin{aligned}
A_{k} & =\left[\begin{array}{cc}
1-R_{s} T_{s} / L_{d} & L_{q} T_{s} \omega_{r}(k) / L_{d} \\
-L_{d} T_{s} \omega_{r}(k) / L_{q} & 1-R_{s} T_{s} / L_{q}
\end{array}\right] \\
B_{k} & =\left[\begin{array}{cc}
T_{s} / L_{d} & 0 \\
0 & T_{s} / L_{q}
\end{array}\right] \\
E_{k} & =\left[\begin{array}{c}
0 \\
-\psi_{f} T_{s} \omega_{r}(k) / L_{q}
\end{array}\right],
\end{aligned}
$$

where $R_{S}$ is the stator resistance, $T_{s}$ is the sampling period, $\omega_{r}$ is the synchronous speed, $L_{d}$ is the $d$-axis inductance, $L_{q}$ is the $q$-axis inductance. In order to compensate for the existence of a delay in the actual system, we need to predict the values corresponding to $k+2$, so eqn (1) is rewritten as

$$
\left[\begin{array}{c}
i_{d}{ }^{p}(k+2) \\
i_{q}{ }^{p}(k+2)
\end{array}\right]=A_{k+1}\left[\begin{array}{c}
i_{d}{ }^{p}(k+1) \\
i_{q}{ }^{p}(k+1)
\end{array}\right]+B_{k+1}\left[\begin{array}{l}
u_{d}(k+1) \\
u_{q}(k+1)
\end{array}\right]+E_{k+1} .
$$

Due to the large mechanical inertia of the system, the approximation is considered:

$$
\omega(k+1)=\omega(k)
$$

Thus the coefficient matrices $A_{k}, B_{k}$ and the back electromotive force $E_{k}$ are approximated as $A_{k+1}=A_{k}, \quad B_{k+1}=B_{k}, \quad E_{k+1}=E_{k}$.

\subsection{Predictive current control strategy}

The cost function is defined according to the control target, and the control input is optimized by the cost function. In order to achieve current tracking control, the cost function can be defined as

$$
J=\left|i_{\text {dref }}-i_{d}{ }^{p}(k+2)\right|^{2}+\left|i_{\text {qref }}-i_{q}{ }^{p}(k+2)\right|^{2},
$$

where: $i_{d r e f}$ and $i_{\text {qref }}$ are the $d$-axis and $q$-axis current references, respectively.

For two-level three-phase inverters, there are 8 possible switching states, corresponding to 6 non-zero voltage vectors and 2 zero voltage vectors. According to eqn (3), each candidate voltage vector will have a corresponding value of the cost function. The smaller the cost function, the smaller the deviation between the predicted current and the reference current. Finally, the voltage vector corresponding to the minimum cost function is selected as the control command sent to the inverter.

The candidate voltage vector selection method used in reference [9] is shown in Fig. 2. In this case, the number of candidate voltage vectors is reduced from 8 to 4 , so the computational burden is reduced. Since switching between non-adjacent voltage vectors is forbidden, at most one bridge arm is active at each sampling period and the switching frequency of the inverter is reduced. 


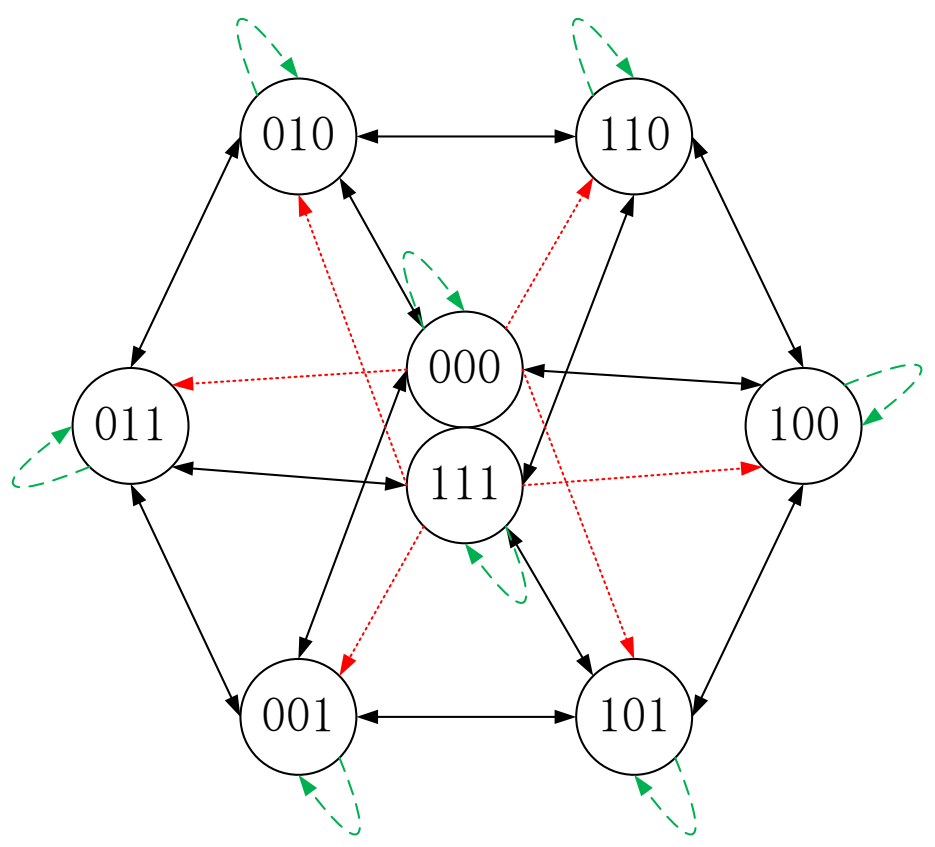

Figure 2: Conventional 4VVs method.

\section{COMMON MODE VOLTAGE SUPPRESSION}

In a two-level three-phase inverter drive system, as shown in Fig. 1, the CMV is defined as $U_{\mathrm{NG}}$, where $U_{\mathrm{NG}}=U_{\mathrm{NO}}+U_{\mathrm{OG}}$. Since $U_{\mathrm{OG}}$ is negligible compared to $U_{\mathrm{NO}}$, the CMV of the three-phase inverter can be expressed as

$$
U_{\mathrm{NG}} \approx U_{\mathrm{NO}}=\left(U_{\mathrm{a}}+U_{\mathrm{b}}+U_{\mathrm{c}}\right) / 3 .
$$

According to eqn (6), the values of the CMV corresponding to all of the 8 voltage vectors can be calculated as is shown in Table 1 . As can be seen from Table 1 , two zero vectors $\left(\boldsymbol{V}_{0}\right.$ and $\left.\boldsymbol{V}_{7}\right)$ corresponding to the CMV amplitude $V_{d c} / 2$, and six non-zero vectors $\left(\boldsymbol{V}_{1} \sim \boldsymbol{V}_{6}\right)$ corresponding to the CMV amplitude $V_{d c} / 6$.

\subsection{Suppression method with non-zero vector control set}

In order to carry out the CMV suppression, reference [10] uses a new voltage vector selection method, as shown in Fig. 3. In this method, the use of zero vectors is forbidden, and a nonadjacent voltage vector is added as the fourth candidate. In this case, the CMV is limited to $V_{\mathrm{dc}} / 6$, but the switching frequency of the inverter is increased due to the use of non-adjacent vectors. It should be pointed out that this kind of operation is one-sidedly pursuing the reduction of CMV while ignoring the drop in current quality.

\subsection{Proposed suppression method with current error limit}

Ensuring good enough current quality is one of the core goals in motor control and should not be overlooked. Therefore, we propose a voltage vector selection method that takes into 
Table 1: Common mode voltage of voltage vectors.

\begin{tabular}{|c|c|c|c|c|}
\hline Voltage vectors & $U_{\mathrm{a}}$ & $U_{\mathrm{b}}$ & $U_{\mathrm{c}}$ & $U_{\mathrm{NG}}$ \\
\hline $\boldsymbol{V}_{0}(0,0,0)$ & $-V_{\mathrm{dc}} / 2$ & $-V_{\mathrm{dc}} / 2$ & $-V_{\mathrm{dc}} / 2$ & $-V_{\mathrm{dc}} / 2$ \\
\hline $\boldsymbol{V}_{1}(1,0,0)$ & $V_{\mathrm{dc}} / 2$ & $-V_{\mathrm{dc}} / 2$ & $-V_{\mathrm{dc}} / 2$ & $-V_{\mathrm{dc}} / 6$ \\
\hline $\boldsymbol{V}_{2}(1,1,0)$ & $V_{\mathrm{dc}} / 2$ & $V_{\mathrm{dc}} / 2$ & $-V_{\mathrm{dc}} / 2$ & $V_{\mathrm{dc}} / 6$ \\
\hline $\boldsymbol{V}_{3}(0,1,0)$ & $-V_{\mathrm{dc}} / 2$ & $V_{\mathrm{dc}} / 2$ & $-V_{\mathrm{dc}} / 2$ & $-V_{\mathrm{dc}} / 6$ \\
\hline $\boldsymbol{V}_{4}(0,1,1)$ & $-V_{\mathrm{dc}} / 2$ & $V_{\mathrm{dc}} / 2$ & $V_{\mathrm{dc}} / 2$ & $V_{\mathrm{dc}} / 6$ \\
\hline $\boldsymbol{V}_{5}(0,0,1)$ & $-V_{\mathrm{dc}} / 2$ & $-V_{\mathrm{dc}} / 2$ & $V_{\mathrm{dc}} / 2$ & $-V_{\mathrm{dc}} / 6$ \\
\hline $\boldsymbol{V}_{6}(1,0,1)$ & $V_{\mathrm{dc}} / 2$ & $-V_{\mathrm{dc}} / 2$ & $V_{\mathrm{dc}} / 2$ & $V_{\mathrm{dc}} / 6$ \\
\hline $\boldsymbol{V}_{7}(1,1,1)$ & $V_{\mathrm{dc}} / 2$ & $V_{\mathrm{dc}} / 2$ & $V_{\mathrm{dc}} / 2$ & $V_{\mathrm{dc}} / 2$ \\
\hline
\end{tabular}

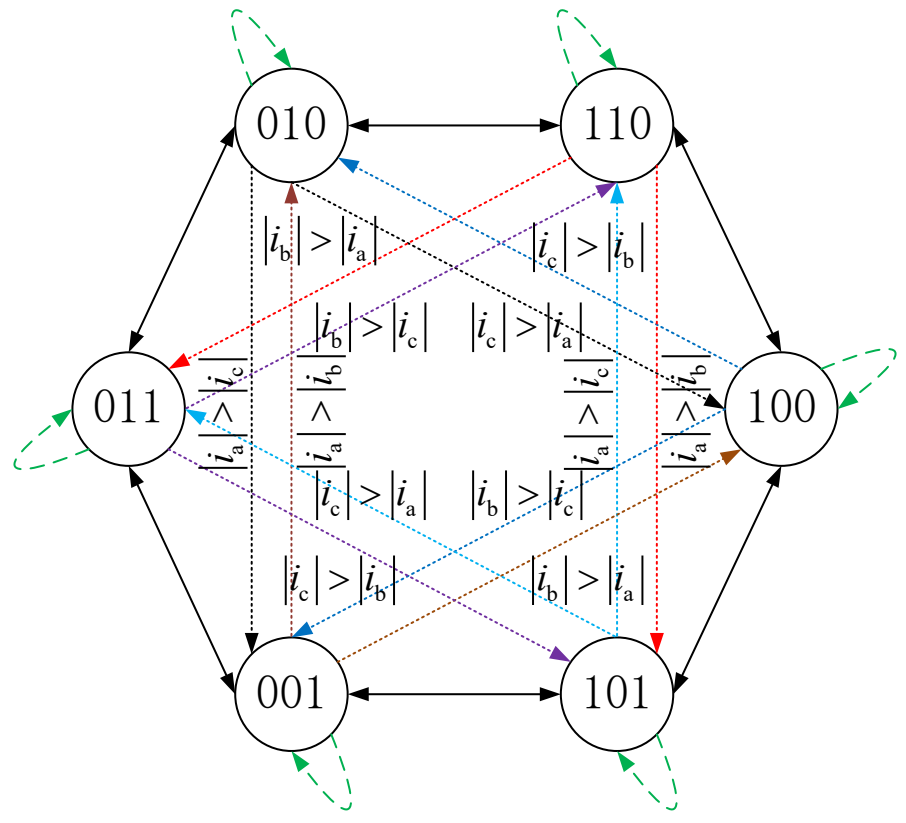

Figure 3: Non-zero 4VVs method [10].

account both current quality and CMV suppression.

In order to keep the switching frequency low, we use only the adjacent voltage and zero vectors, as shown in Fig. 2, but this is not a constant vector selection mode. The difference is that in order to effectively suppress the CMV, we propose to prohibit the use of zero vectors in some special cases. Therefore, the key point of the proposed method is to set an evaluation criterion to dynamically change the selection mode of voltage vectors during the operation of FCS-MPC system. 
In each sampling period, the candidate vector includes three non-zero vectors and one zero vector, so we can get four cost function values $J_{1}, J_{2}, J_{3}$, (calculated by non-zero vectors) and $J_{0}$, (calculated by non-zero vectors). Since the value of the cost function represents the deviation of the predicted current relative to the reference current, we set an error limit $J_{\text {lim. }}$. Then, according to eqn (7) to determine if we need to use zero vectors.

$$
\left\{\begin{array}{lcc}
J_{i}(i=1,2,3) & \text { all }>J_{\lim }, & J_{0}=J_{0} \\
J_{i}(i=1,2,3) & \text { not all }>J_{\lim }, & J_{0} \rightarrow+\infty
\end{array} .\right.
$$

Eqn (7) contains two cases. In the first case, the cost function values for all non-zero vectors are greater than $J_{\lim }$, which means that all of the predicted current deviations exceed the set value. In order to try to ensure better current quality, zero vector will participate in the optimization process as a normal candidate vector. In the second case, the cost function of some non-zero vectors is less than $J_{\lim }$, which means the predicted current deviation is still less than the set value even in the absence of the zero vector. Therefore, the value of cost function of the zero-vector is modified to infinity, which means that the zero vector will be removed during the optimization.

The value of the current error limit $J_{\lim }$ is determined according to the current distortion requirements, and finally the balance between the current precision control and the CMV is reached. Because the absolute value of the current changes greatly during motor operation, it is unwise to set the current error limit to a fixed value. Therefore, the value of $J_{\lim }$ can be set to vary according to current references, as is shown in eqn (8).

$$
J_{\lim }=K^{2}\left(i_{\text {dref }}^{2}+i_{\text {qref }}^{2}\right),
$$

where $K$ is the current relative error limit.

\section{SIMULATION RESULTS}

The simulation results for the high power PMSM drive system will be shown in this section. In order to verify the validity of the CMV suppression method presented in this paper, we established a simulation model of PMSM traction drive system based on MATLAB/ Simulink. The motor used in the simulation was a salient pole PMSM designed for subway vehicles. The DC side voltage of the inverter is $750 \mathrm{~V}$ and the PMSM parameters are shown in Table 2.

In the simulation model, the motor speed is controlled by the mechanical load, and the FCS-MPC system only performs current control. The sampling frequency of FCS-MPC

Table 2: Parameters of the PMSM.

\begin{tabular}{|c|c|}
\hline Parameters & Values \\
\hline Rated current $(\mathrm{A})$ & 160 \\
\hline Stator resistance $(\Omega)$ & 0.078 \\
\hline Inductance in d-axis, $L_{\mathrm{d}}(\mathrm{H})$ & 0.005 \\
\hline Inductance in q-axis, $L_{\mathrm{q}}(\mathrm{H})$ & 0.01 \\
\hline Stator flux $(\mathrm{Wb})$ & 1.35 \\
\hline Pole pairs & 2 \\
\hline
\end{tabular}


system is $10 \mathrm{kHz}$. In order to give full play to the motor torque output capability, current distribution using maximum torque per ampere (MTPA) strategy.

In order to see the validity of the proposed method and its operating characteristics, the total harmonic distortion (THD) of the stator current and the spectrum of the CMV were first tested.

According to eqns (7) and (8), when the relative error limit $K=0$, the voltage vector selection method is consistent with the Conventional 4VVs method which is shown in Fig. 2. When the motor is running at $600 \mathrm{rpm}$, Fig. 4(a) shows the waveform of stator current $\left(i_{\mathrm{a}}\right)$ and CMV. Fig. 4(b) shows the spectrum of the CMV. We can see that the THD of $i_{\mathrm{a}}$ is $3.97 \%$, and the CMV alternates between $V_{\mathrm{dc}} / 2(350 \mathrm{~V})$ and $V_{\mathrm{dc}} / 6(116.7 \mathrm{~V})$.

When the relative error limit $K \neq 0$, the voltage vector selection method will change. That is, both cases described by eqn (7) will occur. Figs 5-7 show the simulation results when $K=15,40$, and 80 , respectively. We can see that values of THD are $4.58 \%, 11.52 \%$, and $16.48 \%$. In other words, the smaller the relative error limit, the better the current quality. However, the larger the relative error limit, the better the CMV suppression effect. As the error limit increases, the average amplitude of the CMV is greatly reduced. This is because zero vectors are rarely used when the relative error margin is large. When $K=80$, it can be seen from Fig. 7 that the zero vector has not been used so the amplitude of CMV is always equal to $V_{\mathrm{dc}} / 6(116.7 \mathrm{~V})$.

For comparison, Fig. 8 shows the simulation results of the Non-zero 4VVs method under the same conditions. We can see that the THD of $i_{\mathrm{a}}$ is $10.31 \%$. Since no zero vector is used, the amplitude of CMV is limited to $V_{\mathrm{dc}} / 6(116.7 \mathrm{~V})$.

Fig. 9 shows the equivalent switching frequency of three different method: the Conventional 4VVs method, the Non-zero 4VVs method, and the proposed method. We can see that the switching frequency of the Non-zero 4VVs method exceeds $2000 \mathrm{~Hz}$, which is significantly higher than the other two methods. Since the proposed method does not use nonadjacent voltage vectors, the switching frequency is not significantly greater than the Conventional 4VVs method, which is about $1600 \mathrm{~Hz}$.

Fig. 10 shows a comparison of the current quality of the three methods. We can see that the Conventional 4VVs method has the best current quality, and the Non-zero 4VVs method greatly increases the current distortion, especially when the current is small. The method proposed in this paper has less current quality than the Conventional 4VVs method due to the CMV rejection. But obviously better than the Non-zero 4VVs method.
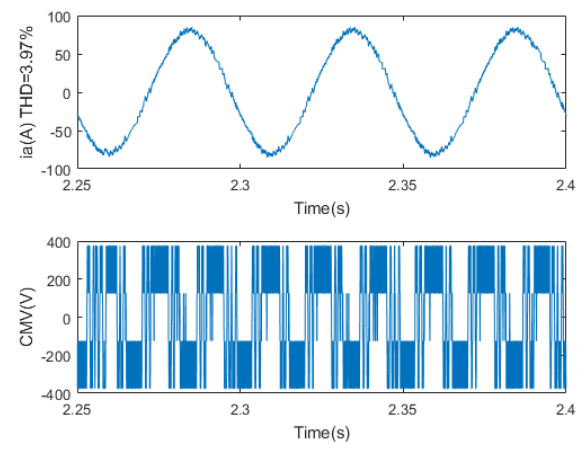

(a)

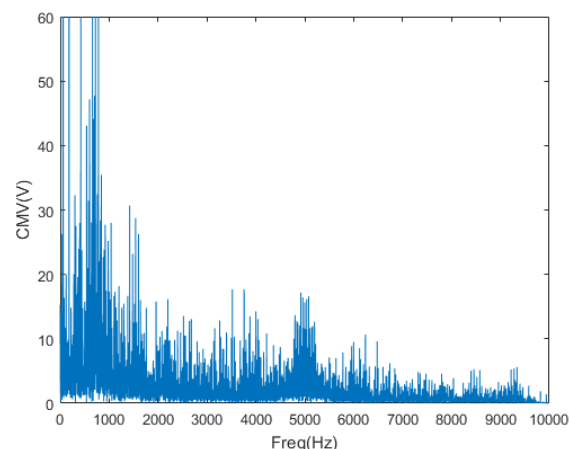

(b)

Figure 4: Conventional 4VVs method (Proposed method, when $K=0$ ). (a) Current of phase A and common mode voltage; (b) Spectrum of common mode voltage. 

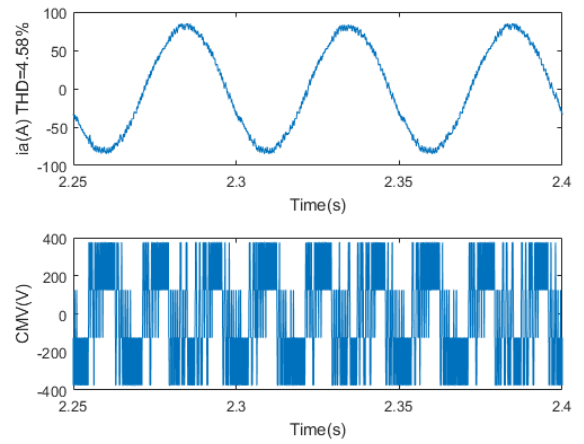

(a)

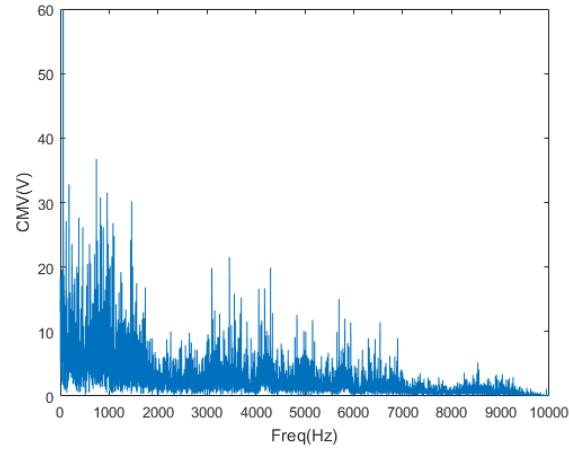

(b)

Figure 5: $\quad$ Proposed method, $K=15$. (a) Current of phase A and common mode voltage; (b) Spectrum of common mode voltage.
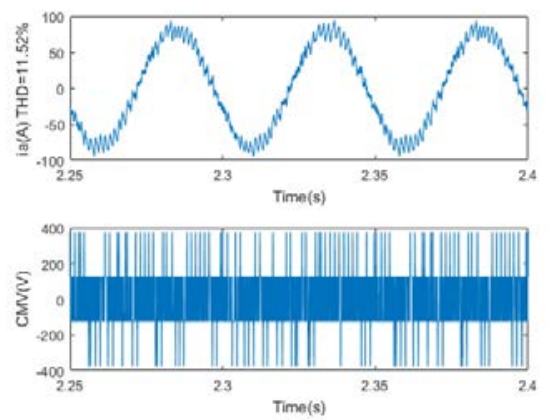

(a)

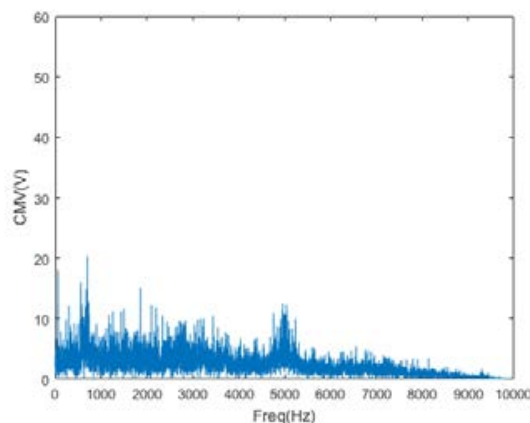

(b)

Figure 6: Proposed method, $K=40$. (a) Current of phase A and common mode voltage; (b) Spectrum of common mode voltage.
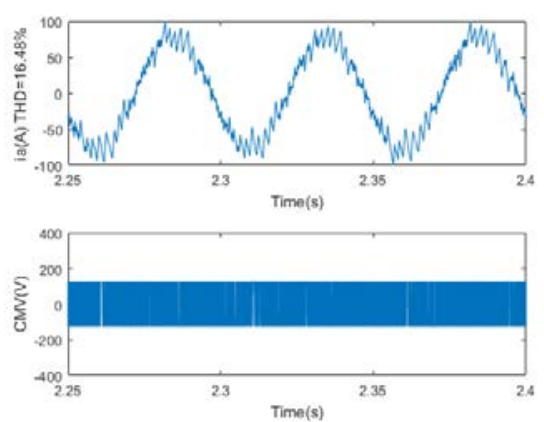

(a)

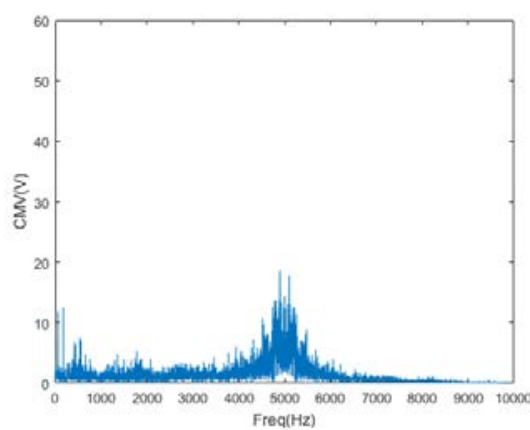

(b)

Figure 7: Proposed method, $K=80$. (a) Current of phase A and common mode voltage; (b) Spectrum of common mode voltage. 

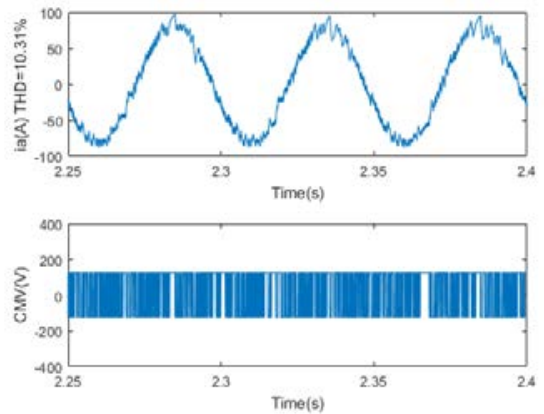

(a)

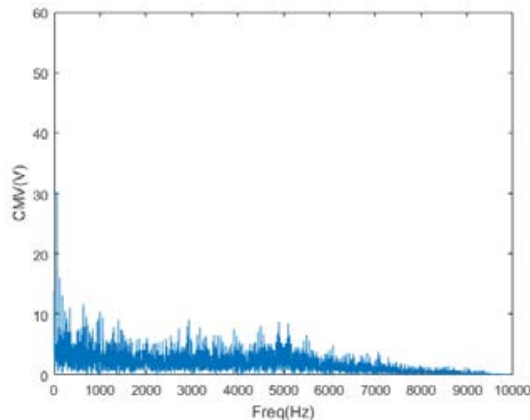

(b)

Figure 8: Non-zero 4VVs. (a) Current of phase A and common mode voltage; (b) Spectrum of common mode voltage.

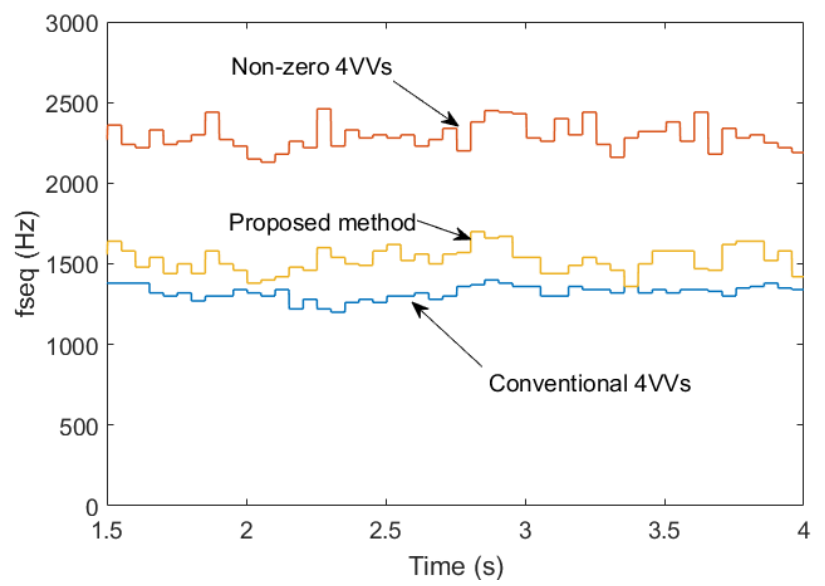

Figure 9: Comparison of the equivalent switching frequency.

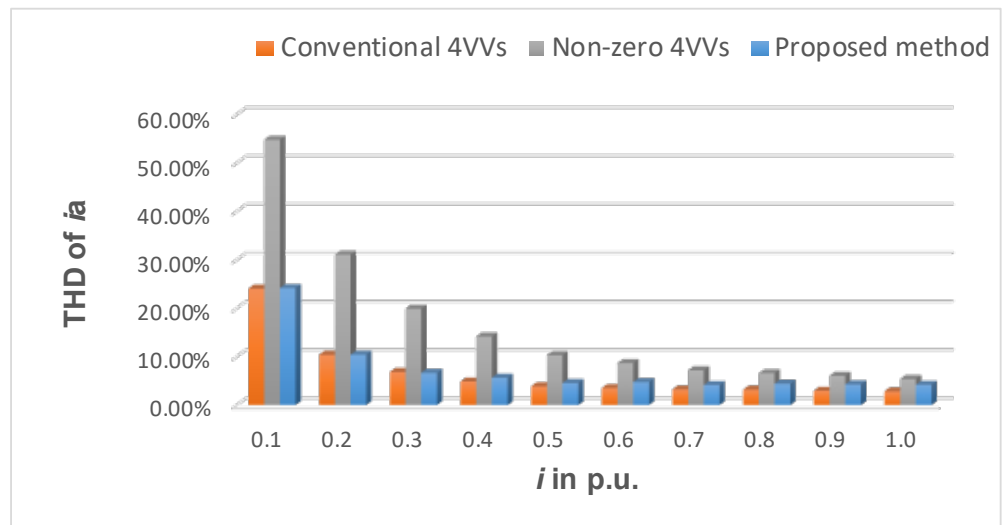

Figure 10: Current THD comparison of three methods. 


\section{CONCLUSION}

Based on FCS-MPC, this paper presents a CMV suppression method considering the current error limit. By comparing with the Conventional 4VVs method and the Non-zero 4VVs method, the proposed method has great advantages in terms of design flexibility and low switching frequency. The great benefit of this method is that it can easily adapt to different applications. By adjusting a single parameter, current quality insurance can be balanced with CMV suppression.

In the further study, we will quantify the influence of current error limits on the current distortion and CMV, and then find the balance between the two aspects.

\section{REFERENCES}

[1] Mei, S. et al., Improved space vector modulation to reduce the common mode voltage for indirect matrix converters. Proceedings of the CSEE, 34(24), pp. 4015-4021, 2014 (in Chinese).

[2] Tallam, R.M. et al., CMV reduction PWM algorithm for AC drives. IEEE Transactions on Industry Applications, 46(5), pp. 1959-1969, 2010.

[3] Cavalcanti, M.C. et al., Modulation techniques to eliminate leakage currents in transformerless three-phase photovoltaic systems. IEEE Transactions on Industrial Electronics, 57(4), pp. 1360-1368, 2010.

[4] Un, E. \& Hava, A.M., A near-state PWM method with reduced switching losses and reduced CMV for three-phase voltage source inverters. IEEE Transactions on Industry Applications, 45(2), pp. 782-793, 2009.

[5] Xueli, Y. et al., Study on suppression strategy of CMV based on predictive control. Colliery Mechanical \& Electrical Technology, (5), pp. 58-64, 2014 (in Chinese).

[6] Mun, S.K. \& Kwak, S., Reducing CMV of three-phase VSIs using the predictive current control method based on reference voltage. Journal of Power Electronics, 15(3), pp. 712-720, 2015.

[7] Hoseini, S.K., Adabi, J. \& Sheikholes-lami, A., Predictive modulation schemes to reduce com-mon-mode voltage in three-phase inverters-fed AC drive systems. IET Power Electronics, 7(4), pp. 840-849, 2014.

[8] Kwak, S. \& Mun, S.K., Model predictive control methods to reduce CMV for threephase voltage source inverters. IEEE Transactions on Power Electronics, 30(9) pp. 5019-5035, 2015.

[9] Preindl, M., Schaltz, E. \& Thøgersen, P., Switching frequency reduction using model predictive direct current control for high-power voltage source inverters. IEEE Transactions on Industrial Electronics, 58(7), pp. 2826-2835, 2011.

[10] Leilei, G. et al., An improved model predictive control based CMV suppression strategy for permanent magnet synchronous generators. Proceedings of the CSEE, 37(16), pp. 4810-4818, 2017 (in Chinese). 